\title{
Polimorfismo enzimático em populações de Melipona quadrifasciata anthidioides Lepeletier (Hymenoptera, Apidae, Meliponinae)
}

\author{
Davi S. Aidar ${ }^{1}$ \\ Rubiane C. Pagotto ${ }^{1}$ \\ Eucleia P.B. Contel ${ }^{1}$
}

\begin{abstract}
Enzymatic polymorphism in Melipona quadrifasciata anthidioides Lepeletier populations (Hymenoptera, Apidae, Meliponinae). Them aim scope of this study is to characterize the enzymatic polymorphism found in the Melipona quadrifasciata Lepeletier, 1936 populations from Ribeirão Preto, São Paulo and Espírito Santo, Brazil and its hybrids. Samples from each colony (about 52) were prepared for starch gel electrophoresis in order to investigate the genetic variation of the following enzimes: esterase (EST), isocitrate dehydrogenase (IDH), malic enzyme (ME), phosphoglucomutase (PGM), superoxide desmutase (SOD), $\alpha$-glycerophosphate dehydrogenase ( $\alpha \mathrm{PGD}$ ), malate dehydrogenase (MDH), leucine aminopeptidase (LAP), hexokinase (HK) and phosphoglucose isomerase (PGI). The analysis showed that LAP and HK did not show enzymatic activity and EST showed two alleles (est-sand and est-f) while all the others were shown to be monomorphic. The allele EST-S showed a frequency of $82,6 \%$.

KEY WORDS. Melipona quadrifasciata anthidioides, polymorphism, electrophoresis, enzyme, stingless bees
\end{abstract}

Pouco se conhece sobre variabilidade proteica em Melipona quadrifasciata Lepeletier, 1936 Os primeiros trabalhos sobre sistemas polimórficos em meliponíneos foram de CONTEL \& MESTRINER (1974), que estudaram 54 colônias de M. subnitida Ducke, 1910, empregando a técnica de eletroforese. Foram identificados três tipos de esterases, esterase 1, esterase 2 e esterase 3 , as quais possuem controle genético independente e polimorfismo em dois locos (EST-2 e EST-3).

A maior parte dos estudos com atividade enzimática em abelhas foi realizada com abelhas Apis (LiMA \& MESTRINER 1985). FALCÃo \& CONTEL (1990) estudaram 10 espécies de meliponíneos e encontraram polimorfismo para a malato desidrogenase e isocitrato desidrogenase em três espécies, também detectou polimorfismo para uma forma de esterase em quatro espécies. YONG (1986) também encontrou polimorfismo nos meliponíneos com relação às enzimas malato desidrogenase (MDH) e isocitrato desidrogenase (IDH). FALCÃo \& CONTEL (1991a) analisando 51 meliponíneos do Amazonas encontraram quatro espécies com polimorfismo para $\mathrm{MDH}$.

1) Departamento de Genética, Faculdade de Medicina de Ribeirão Preto, Universidade de São Paulo. Avenida Bandeirantes 3900, Monte Alegre, 14049-900 Ribeirão Preto, São Paulo, Brasil. 
KeRR \& Krause (1950) e CONTEL \& KeRR (1976) mostraram que em Melipona os acasalamentos ocorrem com apenas um zangão. FALCÃo \& CONTEL (1991a) estudando três colônias de Plebeia droryana Friese, 1900 de Londrina, Paraná, sugeriram que as suas rainhas copularam em $11 \%$ dos casos, com mais de um zangão ou existia mais de uma rainha fisogástrica numa mesma colônia (hipótese considerada menos provável) porque a segregação para MDH e PGM foi de dois tipos de homozigotos em adição ao heterozigoto. PAXTON et al. (1999) constataram que as Melipona e a Scaptotrigona postica Latreille, 1807 acasalam-se 20 e $40 \%$ dos casos, com mais de um macho, respectivamente.

CONTEL \& MESTRINER (1974) propuseram que ocorre monomorfismo em esterases de M. quadrifasciata.

Neste trabalho foi investigado polimorfismo enzimático em população de M. quadrifasciata Lep. por meio da eletroforese em gel de amido para avaliação da variabilidade genética com relação às enzimas: esterase (EST), isocitrato desidrogenase (IDH), enzima málica (ME), fosfoglicomutase (PGM), superóxido dismutase (SOD), $\alpha$-glicerofosfato desidrogenase ( $\alpha \mathrm{PGD}$ ), malato desidrogenase $(\mathrm{MDH})$, leucina aminopeptidase (LAP), hexoquinase (HK) e fosfoglicoisomerase (PGI).

\section{MATERIAL E MÉTODOS}

Cinqüenta e duas colônias de $M$. quadrifasciata, manejadas de acordo com AIDAR (1996), foram amostradas com a coleta de oito operárias de cada uma para serem submetidas a análises eletroforéticas em gel de amido. Dentre as colônias estudadas, parte originaram do Espírito Santo, parte de Ribeirão Preto, São Paulo do Meliponário-A e híbridos entre essas duas populações. As técnicas empregadas para IDH, MDH, EST, ME e LAP foram as mesmas utilizadas por FALCÃO \& CONTEL (1991a); HK, PGI, $\alpha$ PDH, SOD e PGM foram analisadas segundo FALCÃO \& CONTEL (1991b).

As abelhas foram examinadas sob lupa para confirmação do sexo antes de serem submetidas à eletroforese. A confirmação do sexo das abelhas coletadas foi feita mediante a observação das unhas do inseto, ou seja, unhas duplas indicam machos e unhas simples fêmeas. Os sexos também foram diferenciados por meio da observação da presença dos gonóstilos e do tufo de pêlos localizados na extremidade posterior do abdome dos machos.

As estimativas das freqüências gênicas foram feitas utilizando-se o programa Fregen do pacote Genioc (CABELLO \& KRIEGER 1991). O X ${ }^{2}$ de heterogeneidade foi usado na comparação entre as três populações de M. quadrifasciata analisadas, isto é, na população do Meliponário-A, em Ribeirão Preto, na população do Espírito Santo e na população híbrida.

\section{RESULTADOS E DISCUSSÃO}

Foi detectado polimorfismo em M. quadrifasciata em um loco de esterase, complementando os estudos de CONTEL \& MESTRINER (1974) que encontraram monomorfismo para esta enzima numa população desta mesma espécie. 
O loco para esterase apresentou dois alelos denominados Est-S e Est-F, cujos produtos podem ser observados na figura 1. A análise estatística dos dados indicou que as populações de mandaçaia possui freqüências que estão de acordo com o esperado pelo Equilíbrio de Hardy-Weinberg (Tab. I), sendo que após o teste de $\mathrm{X}^{2}$ de heterogeneidade $\left(\mathrm{X}^{2}=1,046 ; \mathrm{gl}=1 ; \mathrm{p}=0,3064\right)$ foi possível considerar as três populações como sendo estatisticamente semelhantes ou homogêneas.

Tabela I. Freqüências alélicas e genotipicas para um loco de esterase em Melipona quadrifasciata nas populações de Ribeirão Preto (RP), Espirito Santo (ES) e Híbridos (RP + ES). (F) Alelo $F$ ("fast"), (FF) homozigoto $F,(G L)$ grau de liberdade, $(P)$ probabilidade, (SF) heterozigoto, (SS) homozigoto S ("slow"), ( $\left.\mathrm{X}^{2}\right)$ teste do Qui-quadrado.

\begin{tabular}{lrrrrrrrrr}
\hline População & Total & SS & SF & FF & S & $F$ & $X^{2}$ & GL & $P$ \\
\hline RP & 346 & 232 & 102 & 12 & $0,817917+0,014594$ & 0,182081 & 0,036 & 1 & 0,8495 \\
ES $\times$ RP & 40 & 29 & 11 & 0 & $0,862507+0,042004$ & 0,137493 & 1,017 & 1 & 0,3132 \\
ES & 17 & 14 & 3 & 0 & $0,911763+0,051174$ & 0,088237 & 0,067 & 1 & 0,7958 \\
\hline
\end{tabular}

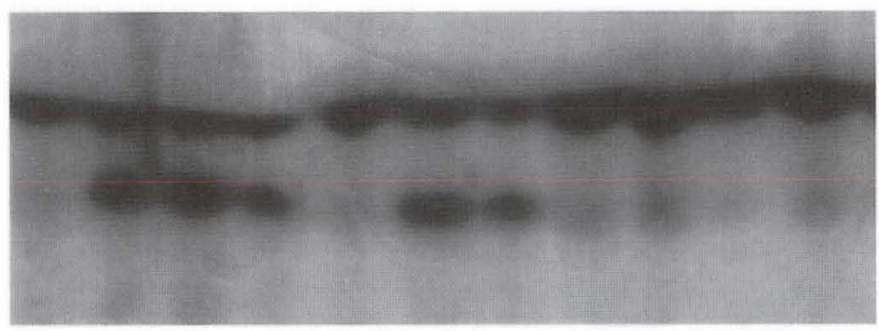

\section{$\begin{array}{lllllllllll}1 & 2 & 3 & 4 & 5 & 6 & 7 & 8 & 9 & 10 & 11\end{array}$}

Fig. 1. Perfil eletroforético das enzimas de esterase de operárias de Melipona quadrifasciata. As amostras 1, 5, 8 a 11 são homozigotas (Est-F/Est-F) e as demais são heterozigotas (Est-F/Est-S).

Foram encontradas operárias homozigotas e heterozigotas, filhas de uma mesma rainha, para esse loco de esterase. Se a rainha copulou com um só macho, isto mostra que ela é obrigatoriamente heterozigota.Caso as operárias da colônia fossem todas homozigotas, seria certo dizer que a rainha teria copulado com um ou mais zangões com o mesmo alelo seu. Por outro lado, sendo todas as operárias heterozigotas, a rainha teria acasalado com um ou mais zangões com alelo diferente do cruzamentos onde foi obtida uma descendência de operárias homozigotas e heterozigotas, a questão era saber se a rainha apresentava homozigose para o mesmo alelo. Caso isso acontecesse, poderia ser afirmado que esta rainha se acasalou com mais de um zangão, sendo pelo menos um deles portador de alelo distinto aos seus.

Quando a rainha acasala-se com um só macho e produz filhas heterozigotas e homozigotas, pode-se inferir que esta rainha é heterozigota, independente do genótipo do zangão. Por outro lado, caso a rainha tenha copulado com vários machos e apresente filhas heterozigotas e filhas homozigotas, nada se pode afirmar quanto ao genótipo desta rainha. 
KERR \& Krause (1950) e CONTEL \& KERR (1976) demostraram que uma rainha de $M$. quadrifasciata pode acasalar apenas uma vez e com um só zangão. Porém, FALCÃo \& CONTEL (199lb) estudando MDH e PGM de $P$. droryana encontraram resultados que sugeriram que, ou as colônias desta espécie podem conter mais de uma rainha em atividade de postura (poliginia) ou que a múltipla fertilização ocorre nessas abelhas. A poliginia nunca foi descrita em M. quadrifasciata e também durante 15 anos de trabalho com Melipona, um dos autores deste trabalho (D.S. Aidar) nunca observou mais de uma rainha fisogástrica numa mesma colônia. Seria mais provável o acasalamento com mais de um zangão e no mínimo dois deles com alelos distintos. Pelos dados apresentados na tabela I pode-se verificar que o loco codificador da EST é polimórfico, sendo que a freqüência do alelo mais raro (EST-F) variou de 8,8 a $18 \%$ nas populações estudadas nas populações amostradas, 26 apresentaram operárias nas quais foram observados os dois alelos.

AGRADECIMENTOS. Agradecemos ao CNPq (Conselho Nacional de Desenvolvimento Científico e Tecnológico) e FAPEMIG (Fundação de Amparo a Pesquisas de Minas Gerais) pelos recursos doados. Ao Prof. Warwick Estevam Kerr, agradecemos pela leitura do manuscrito e pelas sugestões e pela orientação.

\section{REFERÊNCIAS BIBLIOGRÁFICAS}

AIDAR, D.S. 1996. A Mandaçaia: Biologia de abelhas, manejo e multiplicação artifícial de colônias de Melipona quadrifasciata Lep. (Hymenoptera, Apidae, Meliponinae). Brazil. Jour. Genetics, Sér. Monogr., 4: 50-58.

Cabello, P.H. \& H. Krieger. 1991. GENiOC: Programoteca. Rio de Janeiro, Fund. Inst. Oswaldo Cruz.

Contel, E.P.B. \& M.A. Mestriner. 1974. Esterase polymorphism at two in the social bee. Jour. Heredity $65:$ 349-352. . 1974. Esterase polymorphisms at two loci in the social bee. Jour. Hered. 65: 349-352.

CONTEL, E.P.B. \& W.E. KERR. 1976. Origin of males in Melipona subnitida estimated from data of an isozymic polymorphic system. Genetica 46: 271-277.

FALCÃO, T.M.M.A. \& E.P.B. CONTEL. 1990. Genetic variability in natural populations of Brazilian social bees. I. eletrophoretic data for esterases and total proteins. Brazil. Jour. Genet. 13: 731-754.

- 1991a. Genetic variability in natural population of Brazilian social bees. II. Electrophoretic data for PGM and MDH give evidence for multiple fertilization in stingless bees. Rev. Brasil. Genet. 14 (1): 47-59.

$199 \mathrm{lb}$. Genetic variability in natural population of Brazilian social bees. III. Electrophoretic data for ME, GPD,SOD and IDH. Rev. Brasil. Genet. 14 (1): 61-72.

KERR, W.E \& W. KRAUSE. 1950. Contribuição para o conhecimento da bionomia dos Meliponini. Fecundação da rainha em Melipona quadrifasciala Lep. Dusenia, Curitiba, 1 (15): 275-282.

LIMA, L.M.K.S. \& M.A. MESTRINER. 1985. Starch gel eletrophoretic patterns of esterases and nonspecific proteins in 11 different species of Meliponinae bees. Brazil. Jour. Genet. 8: 639-652.

Paxton, R.J.; N. Weibshuh; W. Engls; K. Hartfelder \& J.J.G. Quezada-Euan. 1999. Not only single mating in stingless bees. Naturwissenschaften 86: 143-146.

YoNG, H.S. 1986. Allozime variation in the stingless bee Trigona fuscobalteata (Hymenoptera, Apidae) from peninsular Malaysia. Comp. Biochem. Physiol. 83 (3): 627-628.

Recebido em 29.V1.2000; aceito em 22.V.2001. 